Document downloaded from:

http://hdl.handle.net/10251/78948

This paper must be cited as:

Herrera Tapia, J.; Hernández Orallo, E.; Tomás Domínguez, AE.; Manzoni, P.; Tavares De Araujo Cesariny Calafate, CM.; Cano Escribá, JC. (2016). Improving Message Delivery Performance in Opportunistic Networks using a Forced-stop diffusion scheme. En Ad-hoc, Mobile, and Wireless Networks. Springer. 156-168. doi:10.1007/978-3-319-40509-4_11.

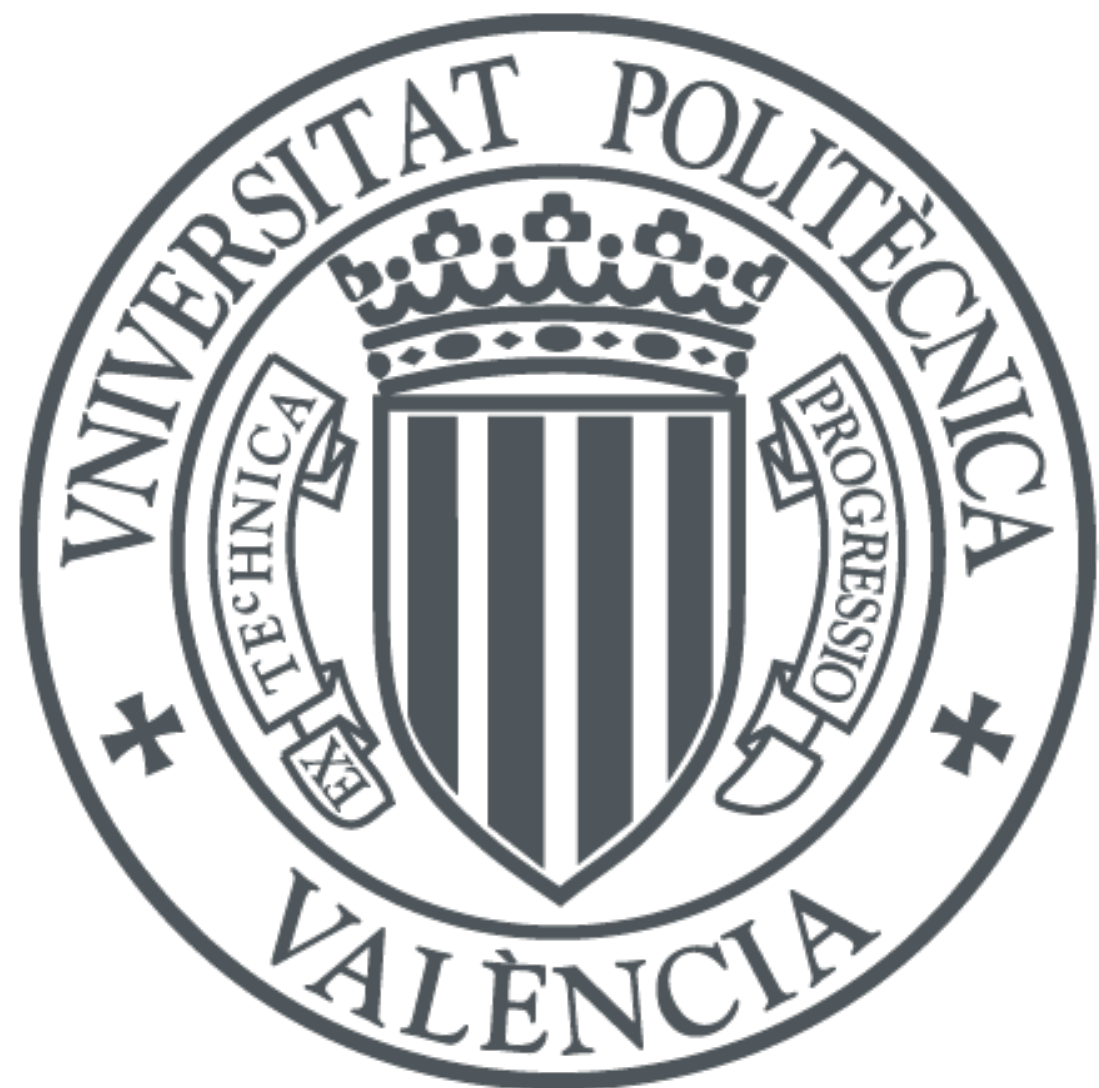

The final publication is available at

https://link.springer.com/chapter/10.1007/978-3-319-40509-4_11

Copyright Springer

Additional Information

The final publication is available at Springer via http://dx.doi.org/10.1007/978-3-319-405094 -11 


\title{
Improving Message Delivery Performance in Opportunistic Networks using a Forced-stop diffusion scheme
}

\author{
Jorge Herrera-Tapia, Enrique Hernández-Orallo, Andrés Tomas, Pietro \\ Manzoni, Carlos T. Calafate, and Juan-Carlos Cano \\ Department of Computer Engineering, \\ Universitat Politècnica de València, \\ Camino de Vera S/N 46022, Valencia, Spain. \\ jorherta@doctor.upv.es, antodo@upv.es, \\ \{ehernandez, pmanzoni, calafate, jucano\}@disca.upv.es
}

\begin{abstract}
The performance of mobile opportunistic networks strongly depends on contact duration. If the contact lasts less than the required transmission times, some messages will not get delivered, and the whole diffusion scheme will be seriously affected.

In this paper we propose a new diffusion method, called Forced-Stop, that is based on controlling node mobility to guarantee a complete message transfer. Using the ONE simulator and realistic mobility traces, we compared our proposal with the classical Epidemic diffusion. We show that Forced-Stop improves the message delivery performance, increasing the delivery ratio up to $30 \%$, and reducing the latency of message delivery up to $40 \%$, with a limited impact on buffer utilisation and message relaying.

These results can be a relevant indication to the designers of opportunistic network applications that could integrate in their products strategies to inform the user about the need to temporarily stop in order to favor the overall data delivery.
\end{abstract}

Keywords: adhoc opportunistic networks, epidemic protocol, DTN, data transfer, wireless adhoc networks

\section{Introduction}

Opportunistic wireless ad-hoc networks $[1,2]$ are an alternative to consider in environments where the wireless infrastructure has become inefficient due to the saturation of requests, or when no communication infrastructure is available. Instead of using the established Internet infrastructure, the communication in mobile opportunistic networks takes place upon the establishment of ephemeral contacts among mobile nodes using direct communication (i.e. Bluetooth or WiFi Direct), and storing the messages in these devices to achieve their full dissemination. 
Considering an epidemic distribution, the duration of the contact time between nodes is probably the key factor in the dissemination of messages. If the contact is too short, the nodes cannot complete the transfer operation, thereby increasing the probability of failure for message dissemination among the nodes in the network.

In this paper we propose a novel messaging diffusion approach, called ForcedStop, that increases the effectiveness of the diffusion process by interacting with the user so as extend the contact duration by making the user wait until the message transmission is complete.

This scheme is already being adopted in some existing short-range messaging protocols such as Apple iOS Airdrop and Google Android Copresence.

We compare the performance of the basic epidemic diffusion and our ForcedStop variation using the ONE (Opportunistic Network Environment) simulator [3]. This simulator was designed and built to specifically evaluate DTN protocols and applications, and focuses on the network layer without considering the details of lower layers such as MAC or physical. In our analysis we have selected a scenario based on a set of human geo-tagged traces obtained during fifteen days, collected by smartphones in the National Chengchi University (NCCU) campus [4]; the message generation patterns (frequency and size) are based on statistics related with social networking applications [5].

The experiments evaluated the dissemination performance of both solutions using different settings, like the buffer sizes or the messages TTL (Time To Live). The results show that the Forced-Stop approach clearly improves the message delivery performance by increasing the delivery success ratio and reducing the delivery time, although extending the message transmission time and introducing some extra overhead in terms of buffer utilisation.

This paper is organised as follows: an overview of related works addressing opportunistic networks and message diffusion is presented in Section 2. The description of our diffusion proposal, experiments and evaluation details are presented in Sections 3 and 4, respectively. Finally, in Section 5, we present some conclusions and future work.

\section{Related works}

Some authors [6] consider opportunistic networks as a subclass of Delay Tolerant Networks (DTNs) [7]. This model is being promoted by the "Internet Research Task Force", and we can find its specifications at http://www.dtnrg.org. Data transmission in DTNs is based on messages or bundles, which are received and then forwarded by nodes. This method is known in networking as Store, Carry and Forward, and it relies on the Bundle Protocol (BP) [8]. Unlike the Internet, in DTNs the information delivery time can increase beyond the minimum required because the communication channels and the data links are intermittent, a phenomenon related to the mobility of transmitters and/or receivers.

Various research works in the literature focussed specifically on message distribution in opportunistic networks. In the case of messages transmitted in social 
networks, the authors of [9] present a detailed analysis with findings from a large scale text messaging study of 70 university students in the United States during four months. In $[10,11]$ the authors examine an utility-based cooperative data dissemination system where the utility of data is defined based on the social relationships between users. There are other proposals, such as [12-16], which evaluate the message dissemination behaviour of the Epidemic protocol by focusing on the mobility patterns of the nodes. In these works, the authors explain the relationship between factors such as speed, mobility model, density of nodes, and places. In addition, some of these authors propose their own mobility model to improve the diffusion in opportunistic networks. There are also some works [17-19] where the authors explain how to improve the dissemination process of epidemic protocols in order to save battery energy. In the case of buffer management, and the influence in the messages dissemination process, some authors [20-22] evaluate the use and optimisations of the buffer trough priority rules to deliver the messages, without performance loss in the information transmission process.

In the context of analytical researches, an analytical model based on Delay Differential Equations is proposed for the authors of [23] to evaluate the diffusion of messages in groups taking into account the transmission time of the messages. This model was validated through simulation. The authors of [24] introduce a mathematical approach for message diffusion in opportunistic networks using the Epidemic protocol.

Summing up, the results of previous papers, highlight the importance of the contact pattern and duration on the message diffusion performance.

\section{Forced-Stop: A new Message Diffusion Approach}

In this section we describe the operations associated to contact-based message diffusion, and we propose a new scheme called Forced-Stop. Moreover, we detail the modifications introduced to the ONE simulator in order to evaluate this type of protocols.

\subsection{Contact Based Message Diffusion Schemes}

The rationale of contact-based messaging is to establish short-range communications between mobile devices. A reference case study could be described as made of various mobile devices provided with a messaging application that notifies and shows to the user any received messages in the subscribed groups. The application is cooperative i.e., it must store the received messages and perform the diffusion of such messages to other nearby nodes. Each node has a limited buffer to store the messages received from other nodes. When two nodes establish a pairwise connection, they exchange any messages stored in their buffers and check whether some of the new messages are suitable for notification to the user. 
Message spreading is based on epidemic diffusion, a concept similar to the spreading of infectious diseases. Basically, when an infected node (i.e., a node that has a message) contacts another node, it infects it (by transmitting the message). Epidemic routing obtains the minimum delivery delay at the expense of increased buffer usage and number of transmissions. A critical factor that determines whether a contact is long enough for a complete transfer of messages is the nodes behaviour when a contact is established. Two variants are possible:

- The nodes continue their movement. In this case, the completion of message transmissions will depend on how long they remain within in communication range. If this contact duration is smaller than the message transmission time, the transmission will fail.

- The nodes stop when they need to exchange information. In this case, the owner of each mobile device will control this exchange by stopping and waiting until the message transmission is completed.

Both approaches raise an interesting question: how much time do contacts last based on a particular mobility model? This clearly depends on average speed and communication range, as detailed in the evaluation section.

In this work we tested the spreading properties of the epidemic protocol by considering both variants of people behaviour to share information. We propose the latter variant to improve the efficiency in data distribution, and we called it Forced-Stop (FS).

\subsection{ONE simulator modifications}

The ONE simulator was designed specifically for evaluating the performance of contact-based dissemination protocols. Among its features are the possibility to generate synthetic traces based on six main DTN routing protocols, and some of their variants, namely: Epidemic, Spray and Wait, Prophet, First Contact, Direct Delivery, and Maxprop. It also provides some relevant mobility models such as Random Walk, Random WayPoint, Linear, and Grid. In addition, it enables using maps and routines based on common user patterns, such as Work and Office Day. Finally, it is easily extendable through the implementation of novel contact-based protocols and mobility models.

Figure 1 shows which modules in ONE were modified. First, in order to implement the Forced-Stop approach, we modified the ActiveRouter, DTNHost, and Connection Java classes. Devices are forced to stop every time they have a message to transmit by setting the speed of both nodes to $0 \mathrm{~m} / \mathrm{s}$. Any stopped device will resume its movement only when the transmission is actually finished.

Moreover, the original ONE message generator, in the MessageEventGenerator class, injects a new message using a random interval time. This random time is uniformly distributed from a range configured in the simulation parameters. In order to get a simulation closer to real human behaviour we modified this module to generate messages following a Poisson process, using an exponential random distribution. 


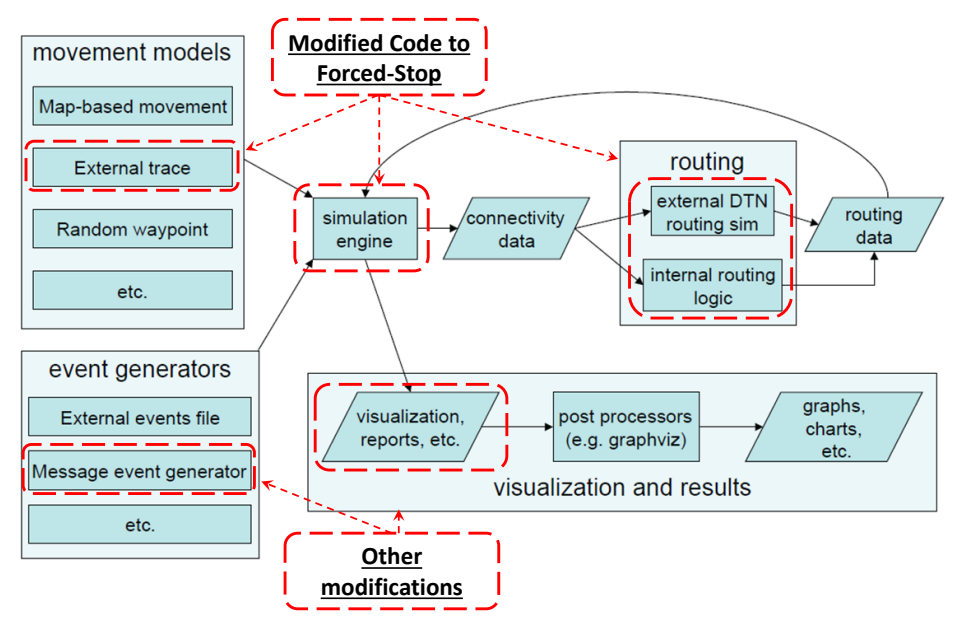

Fig. 1: The red boxes indicate the modified modules in the ONE simulator code. (The figure is based on the original from [3]).

Finally, although the ONE simulator produces a large variety of reports about the simulation process, there was no mechanism to obtain the buffer occupancy. We added a new report class that outputs the average and maximum buffer occupancy of all nodes for each step of the simulation. It also computes the maximum of the average buffer occupancy during the whole simulation.

\section{Performance Evaluation}

The goal of this section is to evaluate the performance of message dissemination under the Epidemic Diffusion and Forced-Stop approaches. The experiments were performed using the ONE simulator, and rely on real human mobility traces from the NCCU University campus. The workload, in terms of message generation is also based on realistic message patterns.

\subsection{Description of Experiments}

The experiments were performed using the ONE simulator with the modifications described in section 3.2 using a real-life movement trace from an experiment at the NCCU University campus [4]. The NCCU Traces were collected using an Android app installed in the smartphones of students belonging to the National Chengchi University. A total of 115 students participated in the experiment. Their GPS data, application usage, Wi-Fi access points, and Bluetooth devices in proximity were recorded over a period of two weeks. Time is specified with a resolution of one second, and the position information is rounded to meters. Figure 2 shows a snapshot of ONE running with the corresponding graphical 
map information. We considered both behaviour models presented previously: classical Epidemic and Forced-Stop (FS).

A key aspect on these experiments is the workload generation. Our generation pattern of messages is related with social networking applications. We considered a typical multimedia messaging application where each user generates messages of different sizes, and shorter messages are far more common than larger ones. Three message sizes were considered: a short text message $(1 \mathrm{kB})$ every hour, a low-resolution picture or photo $(1 M B)$ every 18 hours, and a short video or high resolution picture $(10 \mathrm{MB})$ every 96 hours. These frequencies are loosely based on [5], while sizes are approximations of the typical content produced by current mobile phone hardware. In order to obtain a realistic model of the user behaviour, the interval between messages is generated using an independent Poisson process for each user and message type. Note that this workload is not the same used in [4], so the results presented here can differ from the ones presented in that paper.

Communication range $(r)$ was set to $7.5 \mathrm{~m}$ with bandwidth $B w=2.1 \mathrm{Mb} / \mathrm{s}$, and setup time $T_{s}=0.1 \mathrm{~s}$. These values were selected based on Bluetooth 2.0, Class 2 specifications. Although the maximum range is $10 \mathrm{~m}$, we assume a certain interference, and thereby we reduced the coverage value. Finally, the message Time To Live (TTL) was set to 12 hours or 24 hours, and buffer sizes varied from $50 \mathrm{MB}$ to $1 \mathrm{~GB}$. The main simulation parameters are summarised in Table 1.

Table 1: The main simulation parameters.

\begin{tabular}{|c|c|}
\hline Parameter & Values \\
\hline \hline Buffer Size & $50 \mathrm{MB}, 100 \mathrm{MB}, 200 \mathrm{MB}, 1 \mathrm{~GB}$ \\
\hline Message size & $1 \mathrm{kB}, 1 \mathrm{MB}, 10 \mathrm{MB}$ \\
\hline Routing & Epidemic, Forced-Stop \\
\hline Time to Live & 12 hours, 24 hours \\
\hline
\end{tabular}

\subsection{Evaluation}

In this section we detail the main experiments performed, and discuss the results. We are mainly interested in two performance parameters: the delivery success ratio, that is the ratio of nodes that receive the message, and the delivery latency, that is the time it takes for a message to reach its destination(s). Other key aspects are also studied, such as the overhead, buffer occupancy, and the amount of messages aborted/relayed.

Although there are several alternative diffusion protocols to the Epidemic diffusion (such as the PRoPHET, Spray and Wait, etc.) we compare our approach 


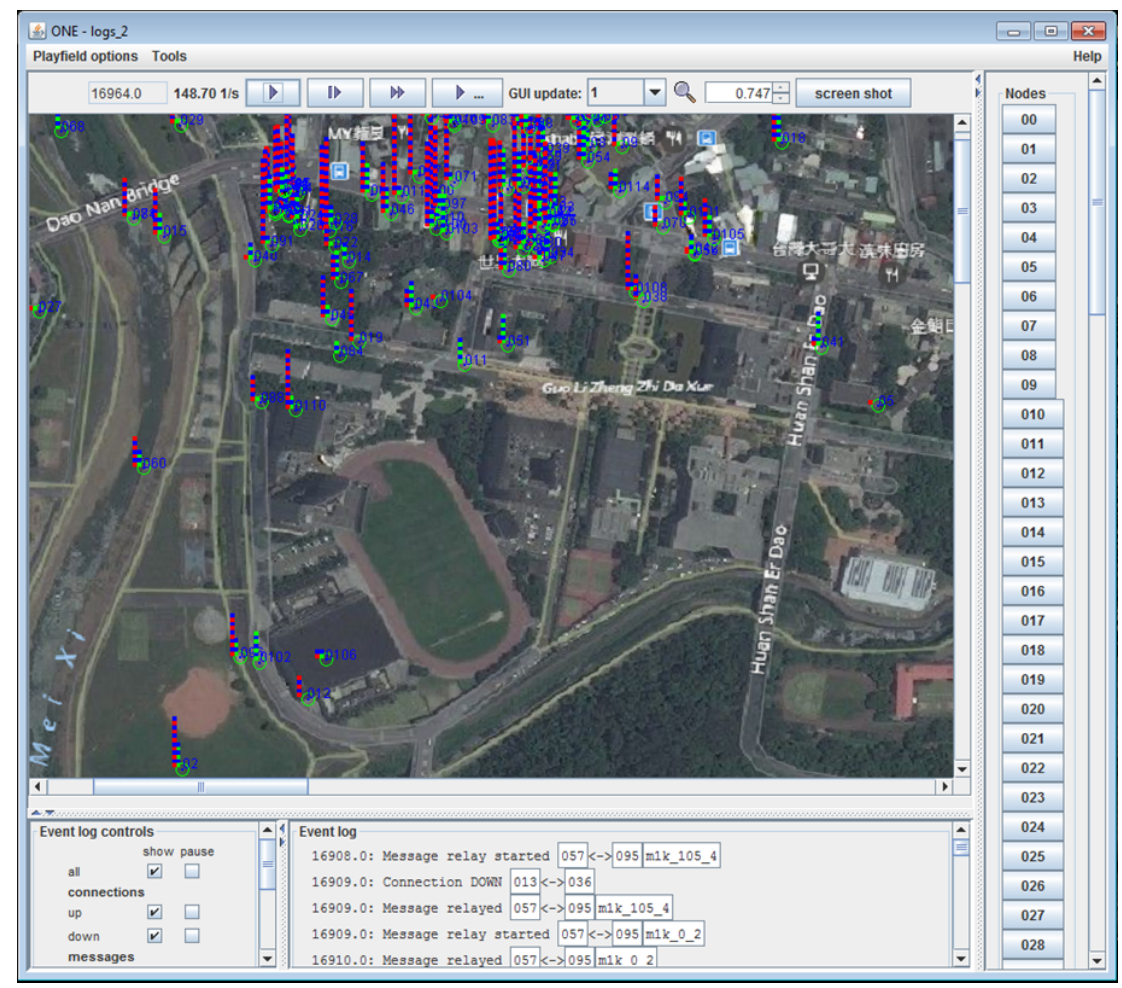

Fig. 2: The ONE simulator running with the NCCU traces.

to the basic Epidemic routing, as it obtains the minimum delivery latency and greatest delivery success ratio. Using the same traces and simulator, the authors of [4] also evaluated the PRoPHET and Spray and Wait protocols, showing that the Epidemic approach obtains the best results at the expense of increased overhead.

We now focus on the dynamics of the diffusion process. Figure 3 shows a sample diffusion graph, using a medium sized $(1 M B)$ message for dissemination. In this figure, each line is a transmission between two nodes showing the time when each transmission was started. Following these transmissions, the message finally arrives to all nodes (a total of 115, including the source).

We now focus on the delivery success ratio. Figure 4 provides a graphical representation of the average delivery success ratio at intervals of 1 hour. This ratio was obtained by calculating the number of messages that are generated at a given hour $h$ of a day: $m s j_{s}[h]$, and the number of these messages that reach their destination $m s j_{r}[h]$; so the hourly ratio is $m s j_{r}[h] / m s j_{s}[h]$. Due to space constrains, we include only two representative plots for the smallest and biggest buffer size $(50 M B$ and $1 G B)$ with different TTLs. In the first plot (Figure 4a) with a TTL of $12 \mathrm{~h}$, we can see that the delivery success ratios are related to 


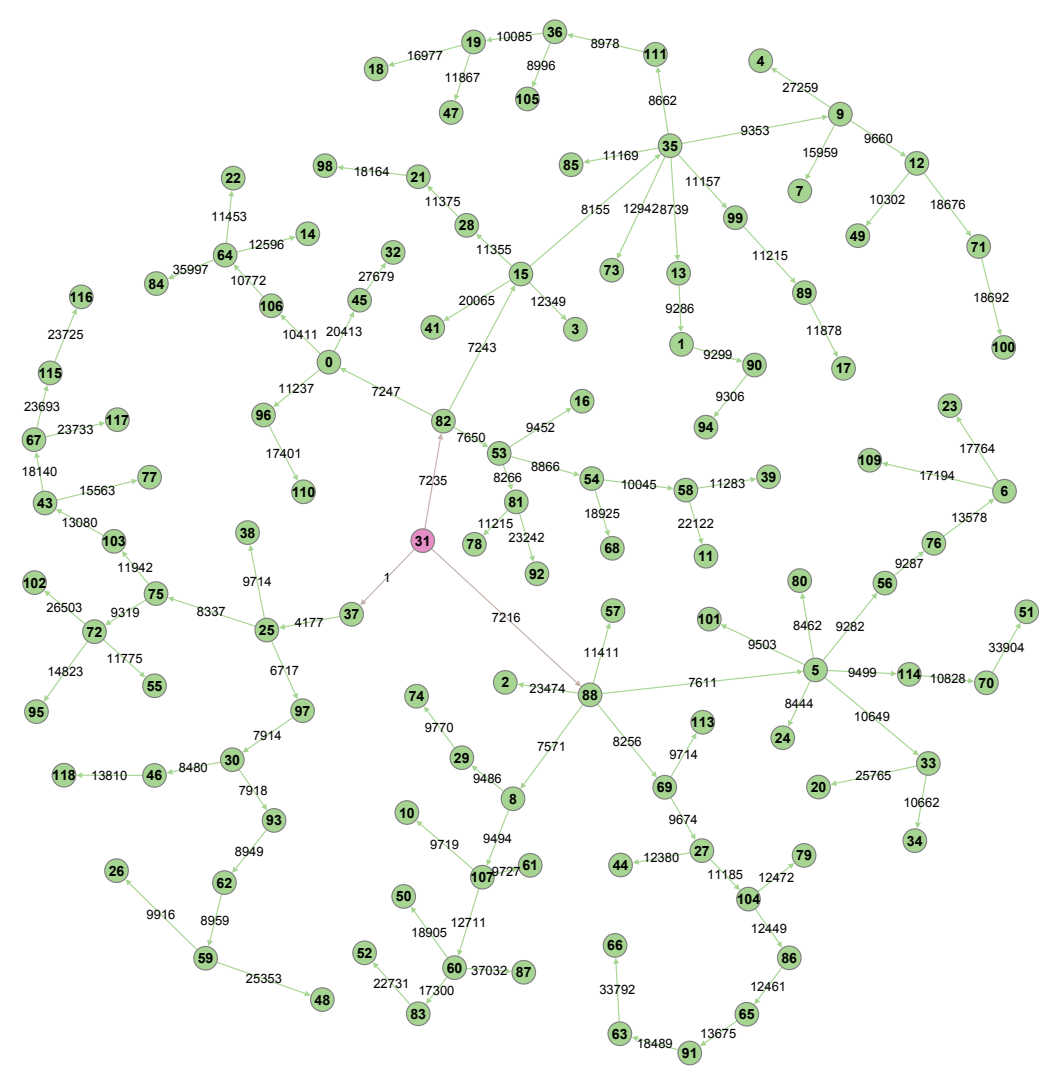

Fig. 3: Message diffusion.

user activities, i.e. at night people are sleeping, thus their motion is reduced and the delivery probability is reduced. At daytime the motion is restored, and the delivery probability goes back to previous levels. Nevertheless, when we increase the TTL to $24 \mathrm{~h}$ (and the buffer size), the results are quite different (see Figure 4a). In this case, due to the longer life time of messages, the daily activities are not so evident, but we can clearly see the weekly activities (for example, days 3-4 and 10-11 are weekends, so message diffusion is reduced). Regarding the effectiveness, in all cases the trend is similar, showing that Forced-Stop has a higher delivery probability than Epidemic diffusion when the rest of parameters remain the same.

Figure 5a shows the average delivery success ratio of all the messages, depending on different buffer sizes for the four combinations of TTL and message spreading approaches simulated. In this case, the ratio is obtained as the quotient between the total number of messages that reach their destination and the 


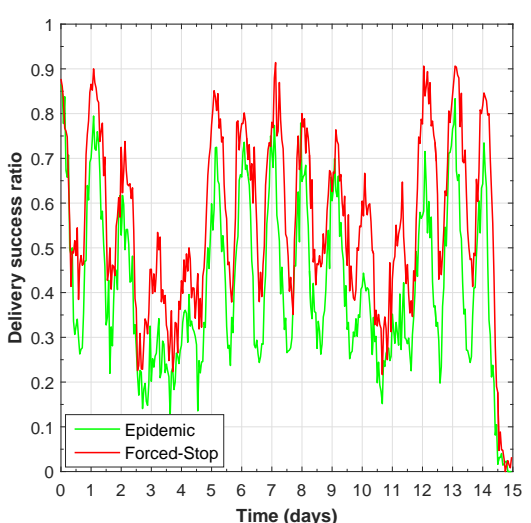

(a) Buffer size $50 \mathrm{MB}$ with $\mathrm{TTL}=12 \mathrm{~h}$

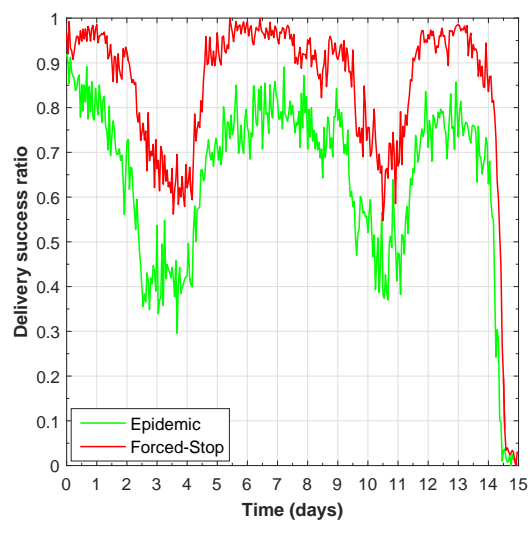

(b) Buffer size $1 \mathrm{~GB}$ with $\mathrm{TTL}=24 \mathrm{~h}$

Fig. 4: Average delivery success ratio by hour.

number of messages generated in the simulation. In this plot we can clearly see that, when the buffer size is bigger, or when the TTL is higher, more messages are stored in each node, improving the delivery probability. We can see that the Forced-Stop approach, as it avoids incomplete transmissions, improves the delivery probability. The Forced-Stop approach presents approximately a 30\% higher ratio than for the Epidemic protocol. This advantage is even higher for a TTL of 24 hours. The most interesting result from these experiments is that buffer size is not so determinant after a certain value, since it is sufficiently large to store most of the generated messages.

Regarding the message delivery latency, Figure $5 \mathrm{~b}$ shows the average delivery time of all messages depending on buffer size, for the two different dissemination approaches and TTL times. In general, using Forced-Stop reduces the latency time in contrast to the Epidemic protocol, decreasing by about $20 \%$ or $30 \%$ for a TTL of 12 or 24 hours. The impact of buffer size is not as dramatic, being close to $5 \%$ or $10 \%$ for each TTL, as it is the case for the delivery probability. Note also that the latency increases with the TTL, due to the improved delivery ratio, i.e., more messages reach the destination, but with greater latency.

Considering the overhead of the protocols, Figure 6 compares the buffer occupancy (that is, the percentage of buffer used), for both dissemination approaches. These plots show the average buffer occupancy among all nodes at each time step in the simulation. As in previous experiment, and, due to space constrains, we only include two extreme cases, 50MB and 1GB buffer size, with a TTL 


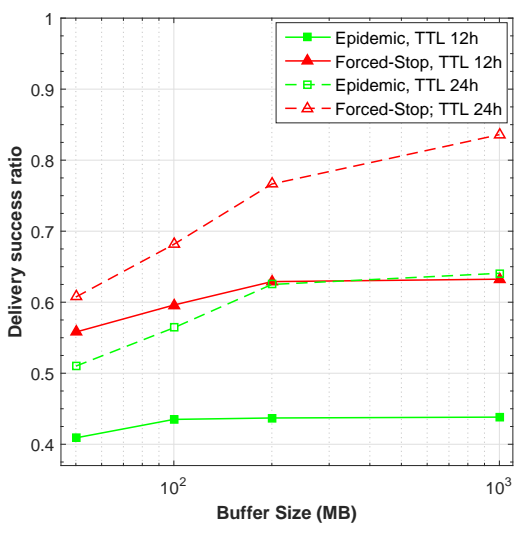

(a) Average delivery success ratio.

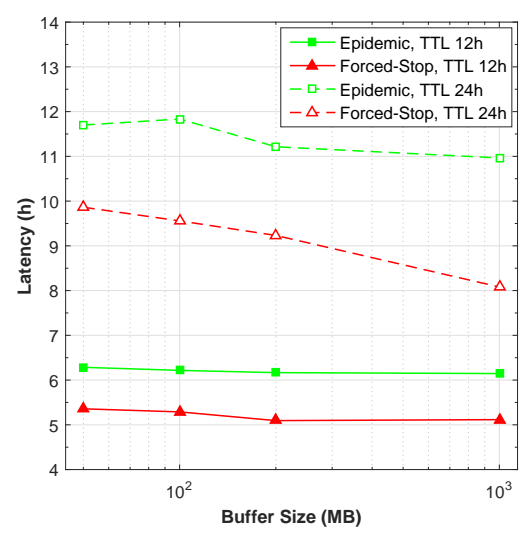

(b) Delivery latency time (in hours).

Fig. 5: Delivery success ratio and Latency depending on buffer size (with x-axis in $\log$ scale)

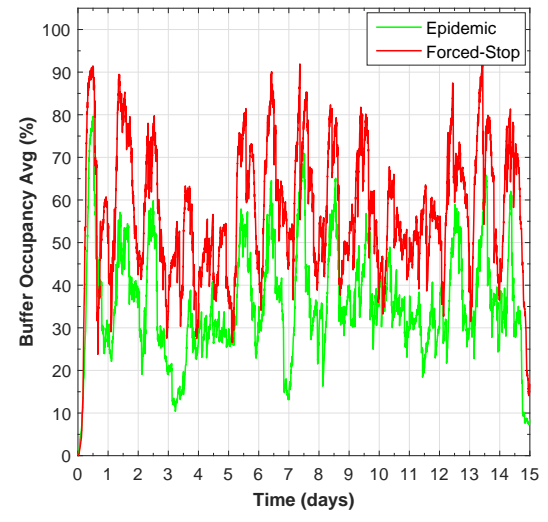

(a) Buffer size 50MB, TTL 12h.

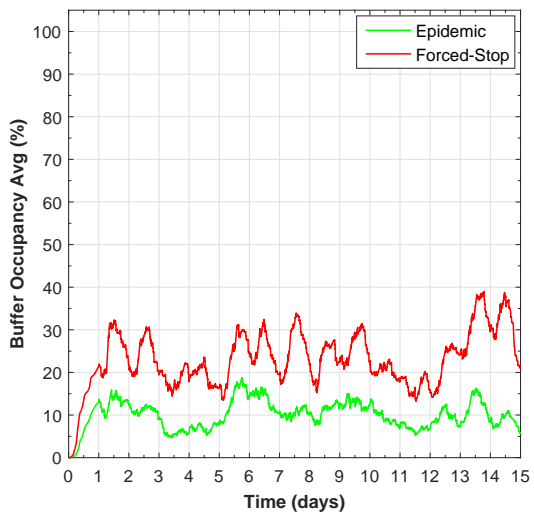

(b) Buffer size 1GB, TTL 24h.

Fig. 6: Buffer occupancy over simulation time.

value of 12 and 24 hours, respectively. From these results it becomes clear that a buffer of $1 \mathrm{~GB}$ is large enough to store all messages even for a large TTL, while a buffer of 50MB gets easily full even for a smaller TTL. In order to determine the required buffer size, we evaluate the maximum buffer occupancy for the whole simulation. Figure 7a plots this maximum buffer occupancy for different TTL values and dissemination approaches. As expected, buffer occupancy is higher for 


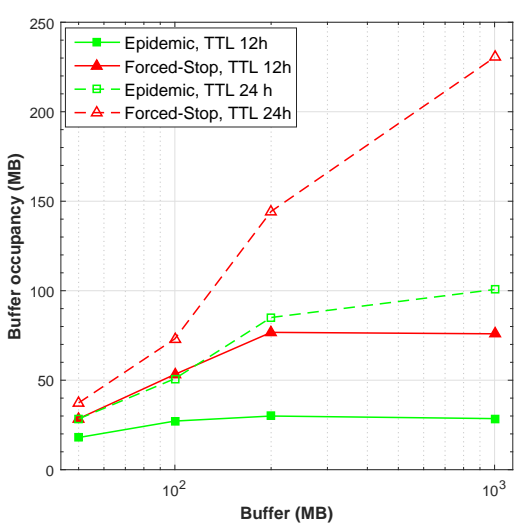

(a) Maximum buffer occupancy.

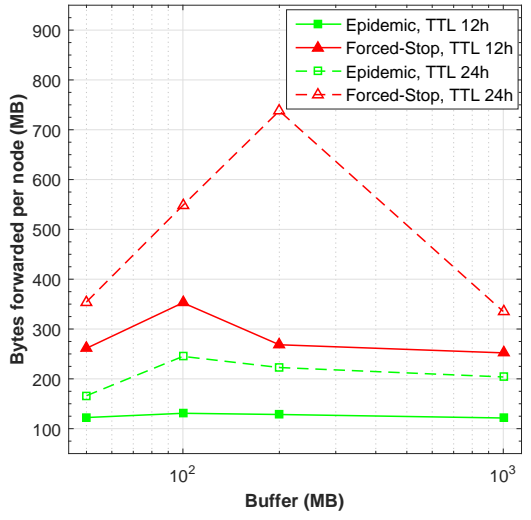

(b) Bytes daily forwarded per node.

Fig. 7: Overhead results: Buffer occupancy and forwarded bytes

the Forced-Stop than for the Epidemic diffusion scheme because, as the delivery probability is higher, more messages remain alive in the whole network.

Another interesting metric, related to protocol overhead, is the amount of information forwarded per node. Similarly to the previous analysis, the results in Figure 7b are grouped by TTL. As the delivery probability increases, the amount of data delivered increases until the buffer size is big enough to hold most messages (200MB for a 12 hours TTL, and 1GB for 24 hours). This effect is caused by the dissemination process, where messages are only sent if the receiver does not have them. If the buffer is not large enough, a lot of messages are discarded to make room for new messages, and those discarded messages are sent again when nodes contact for a second time.

Table 2: Message statistics with a TTL of $12 \mathrm{~h}$ (x 1,000).

\begin{tabular}{|c|c|c|c|c|r|r|r|c|}
\hline $\begin{array}{c}\text { Buffer } \\
\text { size }\end{array}$ & Protocol & Created & Relayed & Aborted & Dropped & Removed & Delivered & $\begin{array}{c}\text { Delivery } \\
\text { probability }\end{array}$ \\
\hline \hline \multirow{2}{*}{$50 \mathrm{MB}$} & Epidemic & 44.2 & $2,154.9$ & 7.1 & $1,480.9$ & 696.5 & 18.1 & 0.41 \\
\cline { 2 - 9 } & Forced-Stop & 44.1 & 6.065 .2 & 0.0 & $2,665.8$ & $3,409.7$ & 24.6 & 0.56 \\
\hline \multirow{2}{*}{$100 \mathrm{MB}$} & Epidemic & 44.2 & $1,793.1$ & 7.8 & $1,677.6$ & 136,5 & 19.2 & 0.44 \\
\cline { 2 - 9 } & Forced-Stop & 44.0 & $4,967.6$ & 0.0 & $2,886.8$ & $2,089.8$ & 26,1 & 0.60 \\
\hline \multirow{2}{*}{$200 \mathrm{MB}$} & Epidemic & 44.3 & $1,690.9$ & 7,7 & $1,712.0$ & 0.0 & 19.3 & 0.44 \\
\cline { 2 - 8 } & Forced-Stop & 44.4 & $3,272.1$ & 0.0 & $3,151.6$ & 128.2 & 27.9 & 0.63 \\
\hline \multirow{2}{*}{$1 \mathrm{~GB}$} & Epidemic & 43.9 & $1,701.5$ & 7.5 & $1,722.2$ & 0.0 & 19.2 & 0.44 \\
\cline { 2 - 8 } & Forced-Stop & 44.3 & $3,166.8$ & 0.0 & $3,174.3$ & 0.0 & 28.1 & 0.63 \\
\hline
\end{tabular}


Finally, we present the results obtained related to the message dynamics, that is, the number of messages relayed, dropped and aborted. When mandatory stops are not enforced, transmissions will depend on the duration of contacts. If a transmission cannot be completed for the duration of a given contact, it is considered an aborted transmission. Also, a message could be dropped if the TTL expires, and removed to make room for new messages when the buffer is full. Tables 2 and 3 show the message count for all simulations considered, also showing the number of messages created, relayed and delivered. As expected, there are no aborted messages using the Forced-Stop approach this number comparatively small when using the Epidemic approach. Although this number is not large by any means, the effect of not losing those transmissions is quite noticeable in the delivery probability. In the Force-Stop approach there are more relayed, dropped and removed messages than in the regular Epidemic approach, simply because buffer occupancy is higher. As there are more messages in the buffers, more messages are transmitted, and so more message could be dropped or removed.

Table 3: Message statistics with a TTL of $24 \mathrm{~h}$ (x 1,000).

\begin{tabular}{|c|c|c|c|c|c|c|c|c|}
\hline $\begin{array}{c}\text { Buffer } \\
\text { size }\end{array}$ & Protocol & Created & Relayed & Aborted & Dropped & Removed & Delivered & $\begin{array}{c}\text { Delivery } \\
\text { probability }\end{array}$ \\
\hline \hline \multirow{2}{*}{$50 \mathrm{MB}$} & Epidemic & 44.2 & $4,088.9$ & 7.5 & $1,525.2$ & $2,561.4$ & 22.6 & 0.51 \\
\cline { 2 - 9 } & Forced-Stop & 44.5 & $13,522.1$ & 0.0 & $2,604.5$ & $10,881.0$ & 27,0 & 0.61 \\
\hline \multirow{2}{*}{$100 \mathrm{MB}$} & Epidemic & 44.4 & $3,846.4$ & 9,5 & $1,854.7$ & $1,979.7$ & 25.1 & 0.60 \\
\cline { 2 - 9 } & Forced-Stop & 44.0 & $13,048.5$ & 0.0 & $3,064.2$ & $9,906.6$ & 29.9 & 0.68 \\
\hline \multirow{2}{*}{$200 \mathrm{MB}$} & Epidemic & 44.2 & $2,969.3$ & 9.3 & $2,385.3$ & 564.8 & 27.7 & 0.63 \\
\cline { 2 - 9 } & Forced-Stop & 44.1 & $11,137.0$ & 0.0 & $3,581.6$ & $7,444.6$ & 33.8 & 0.77 \\
\hline \multirow{2}{*}{$1 \mathrm{~GB}$} & Epidemic & 44.2 & $2,541.8$ & 9.2 & $2,513.1$ & 0.0 & 28.3 & 0.64 \\
\cline { 2 - 9 } & Forced-Stop & 44.3 & $4,183.0$ & 0.0 & $4,065.8$ & 0.0 & 37.0 & 0.84 \\
\hline
\end{tabular}

\section{Conclusions and future work}

This paper presented a new diffusion method, called Forced-Stop, based on controlling nodes mobility to achieve complete message transfers in opportunistic networks. Using the ONE simulator and a realistic environment based on real human mobility traces, we compared our proposal with the classical Epidemic diffusion.

Our experiments showed that forcing devices to stop moving to complete the data delivery process can improve the performance of the whole diffusion process. Our diffusion model provides a higher delivery success ratio and lower delivery times at the expense of higher buffer occupation and longer transmission.

These results can be a relevant indication to the designers of opportunistic network applications that could integrate in their products strategies to inform the user about the need to temporarily stop in order to increase the overall data 
delivery. Our interest in this type of analysis, on the long term, is focused on the design of cross-layer content distribution strategy to improve information sharing in opportunistic networks, and to provide a clear insight on how to develop and deploy efficient cooperative applications.

\section{Acknowledgment}

This work was partially supported by the Ministerio de Economía y Competitividad, Programa Estatal de Investigación, Desarrollo e Innovación Orientada a los Retos de la Sociedad, Proyectos I+D+I 2014, Spain, under Grant TEC201452690-R, the Generalitat Valenciana, Spain, under Grant AICO/2015/108, the Secretaría Nacional de Educación Superior, Ciencia, Tecnología e Innovación del Ecuador (SENESCYT), and the Universidad Laica Eloy Alfaro de Manabí, Ecuador.

\section{References}

1. L. Pelusi, A. Passarella, and M. Conti, "Opportunistic Networking: Data Forwarding in Disconnected Mobile Ad Hoc Networks," IEEE Communications Magazine, pp. 134-141, 2006

2. S. Ferretti, "Shaping opportunistic networks," Computer Communications, vol. 36, pp. 481-503, 2013.

3. A. Keränen, J. Ott, and T. Kärkkäinen, "The ONE simulator for DTN protocol evaluation," Proceedings of the Second International ICST Conference on Simulation Tools and Techniques, Rome, Italy, 2009.

4. T.-C. Tsai and H.-H. Chan, "NCCU Trace: social-network-aware mobility trace," Communications Magazine, IEEE, vol. 53, pp. 144-149, 2015.

5. http://www.statista.com/chart/1938/monthly-whatsapp-usage-per-user, "An Average WhatsApp User Sends Messages per Month," 15/09/2015.

6. J. Niu, J. Guo, Q. Cai, N. Sadeh, and S. Guo, "Predict and Spread: an Efficient Routing Algorithm for Opportunistic Networking," Wireless Communications and Networking Conference (WCNC), 2011 IEEE, pp. 498-503, Cancún, México, 2011.

7. G. S. Thakur, U. Kumar, A. Helmy, and W.-J. Hsu, "On the efficacy of mobility modeling for DTN evaluation: Analysis of encounter statistics and spatiotemporal preferences," Wireless Communications and Mobile Computing Conference (IWCMC), 2011 7th International, pp. 510-515, Istanbul, Turkey, 2011.

8. F. Warthman, "Delay-and Disruption-Tolerant Networks (DTNs) A Tutorial, version 2.0," The InterPlaNetary (IPN) Internet Project. InterPlanetary Networking Special Interest Group (IPNSIG), 2012.

9. A. Battestini, V. Setlur, and T. Sohn, "A Large Scale Study of Text Messaging Use," 12th International conference on Human computer interaction with mobile devices and services MobileHCI, pp. 1-10, Lisbon, Portugal, 2010.

10. A. Förster, K. Garg, H. A. Nguyen, and S. Giordano, "On context awareness and social distance in human mobility traces," Third ACM International Workshop on Mobile Opportunistic Networks, pp. 5-12, Zürich, Switzerland, 2012.

11. C. Boldrini, M. Conti, and A. Passarella, "Modelling Data Dissemination in Opportunistic Networks," Proceedings of the third ACM workshop on Challenged networks - CHANTS '08, pp. 89-96, San Francisco, USA, 2008. 
12. E. Natalizio and V. Loscrí, "Controlled mobility in mobile sensor networks: Advantages, issues and challenges," Telecommunication Systems, vol. 52, no. 4, pp. 2411$2418,2013$.

13. V. V. Neena and V. M. A. Rajam, "Performance analysis of epidemic routing protocol for opportunistic networks in different mobility patterns," 2013 International Conference on Computer Communication and Informatics, pp. 1-5, Coimbatore, India, 2013.

14. N. Mehta and M. Shah, "Performance Evaluation of Efficient Routing Protocols in Delay Tolerant Network under Different Human Mobility Models," vol. 8, pp. 169$178,2015$.

15. J. Su, A. Chin, A. Popivanova, A. Goel, and E. D. Lara, "User Mobility for Opportunistic Ad-Hoc Networking," Sixth IEEE Workshop on Mobile Computing Systems and Applications (WMCSA 2004), Low Wood, United Kingdom, 2004.

16. Z. Feng and K.-W. Chin, "A Unified Study of Epidemic Routing Protocols and their Enhancements," Parallel and Distributed Processing Symposium Workshops PhD Forum (IPDPSW), 2012 IEEE 26th International, pp. 1484-1493, Shanghai, China, 2012.

17. D. Vardalis and V. Tsaoussidis, "Exploiting the potential of DTN for energyefficient internetworking," Journal of Systems and Software, vol. 90, pp. 91-103, 2014.

18. F. D. Rango, S. Amelio, and P. Fazio, "Epidemic Strategies in Delay Tolerant Networks from an Energetic Point of View : Main Issues and Performance Evaluation," Journal of Networks, 2012.

19. J. Herrera-Tapia, P. Manzoni, E. Hernández-Orallo, C. T. Calafate, and J.-C. Cano, "Power Consumption Evaluation in Vehicular Opportunistic Networks," IEEE 12th CCNC 2015 Workshops - VENITS, pp. 925-930, Las Vegas, USA, 2015.

20. V. Erramilli and M. Crovella, "Forwarding in opportunistic networks with resource constraints," Proceedings of the third ACM workshop on Challenged networks CHANTS '08, pp. 41-47, San Francisco, USA, 2008.

21. G. Fathima and R. Wahidabanu, "Buffer management for preferential delivery in opportunistic delay tolerant networks," International Journal of Wireless and Mobile Networks (IJWMN), vol. 3, pp. 15-28, 2011.

22. D. Pan, Z. Ruan, N. Zhou, X. Liu, and Z. Song, "A comprehensive-integrated buffer management strategy for opportunistic networks," EURASIP Journal on Wireless Communications and Networking, pp. 1-10, 2013.

23. E. Hernández-Orallo, J. Herrera-Tapia, J.-C. Cano, C. T. Calafate, and P. Manzoni, "Evaluating the Impact of Data Transfer Time in Contact-Based Messaging Applications," IEEE Communications Letters, vol. 19, pp. 1814-1817, 2015.

24. C. S. de Abreu and R. M. Salles, "Modeling message diffusion in epidemical DTN," Ad Hoc Networks, vol. 16, pp. 197-209, Benidorm, Spain, 2014. 